\title{
NOVO SEPARADOR E CLASSIFICADOR MAGNÉTICO: APLICAÇÕES EM PROCESSAMENTO DE MINÉRIOS
}

\author{
P. A. Augusto ${ }^{1, a}$, T. Castelo-Grande ${ }^{1, b}$, P. Augusto $^{2}$, D. Barbosa ${ }^{1, c}$ \\ ${ }^{1}$ Departamento de Engenharia Química - Faculdade de Engenharia da Universidade do \\ Porto. Rua Dr. Roberto Frias, s/n. 4200-465, Porto- PORTUGAL. \\ a) paa@fe.up.pt ; b) tcg@fe.up.pt ; c) dbarbosa@fe.up.pt ; \\ ${ }^{2}$ Departamento de Matemática e Engenharias - Universidade da Madeira. Caminho da \\ Penteada. Funchal - PORTUGAL \\ augusto@uma.pt
}

Trabalho apresentado no XXI ENTMME, Natal-RN, novembro, 2005

\section{RESUMO}

Os métodos convencionais de separação magnética, separam as partículas desviando-as da corrente principal ou coleccionando-as em matrizes. No entanto nenhum deste métodos convencionais apresenta a característica de classificar as partículas enquanto elas são separadas. O novo separador-classificador magnético desenvolvido, para além da apresentação de um novo processo de separação, preenche esta lacuna. Este processo de separação-classificação é conseguido através de uma geometria inovadora aplicada a uma configuração também ela inovadora.

É feita uma breve análise dos separadores magnéticos actualmente mais utilizados na indústria e seus resultados genéricos em termos de classificação de partículas magnéticas.O novo separador-classificador é apresentado e os seus princípios de operação descritos. São destacadas duas principais aplicações do novo separador-classificador: a separação e classificação de materiais paramagnéticos com a mesma natureza (constituição química) mas com diferentes graus de pureza, e a separação-classificação de materiais paramagnéticos segundo a sua natureza (constituição química). São ainda discutidas outras aplicações práticas possíveis para o novo separador-classificador magnético.

PALAVRAS-CHAVE: separação magnética; classificação magnética; processamento de minérios, materiais paramagnéticos. 


\section{NOVO SEPARADOR E CLASSIFICADOR MAGNÉTICO: APLICAÇÕES EM PROCESSAMENTO DE MINÉRIOS}

\section{INTRODUÇÃO}

A separação magnética de minérios constitui uma área bem desenvolvida: vários separadores magnéticos foram desenhados e construídos e várias patentes foram registadas contemplando os mais variados aparelhos separadores. Deste modo pode afirmar-se que hoje em dia a separação magnética de partículas magnéticas é conseguida com grande precisão.

Verifica-se, no entanto, que os modelos com aplicação prática industrial se podem dividir em dois principais ramos: os separadores magnéticos que atingem os objectivos de separação desviando as partículas magnéticas da corrente principal - e.g. separação magnética de gradiente livre -, e os separadores magnéticos que atingem os mesmos objectivos de separação coleccionando as partículas magnéticas em matrizes ferromagnéticas - e.g. separação magnética de alto gradiente.

Isto quer dizer que nenhum dos modelos de separação magnética desenvolvidos até ao momento apresenta a característica de classificar diferencialmente as partículas enquanto elas são separadas (Augusto e Martins, 2000a). De facto, verifica-se invariavelmente que os separadores magnéticos actuais são apenas capazes, numa só passagem, de separar o fluxo alimentado em três classes (Figura 1): a classe das partículas magnéticas, a classe dos mistos e a classe das partículas não magnéticas. Para se obter uma classificação mais refinada das partículas magnéticas teremos de efectuar várias passagens no mesmo separador, ou alternativamente utilizar os chamados sistemas em cascata (Figura 2).

Deste modo, verificava-se a falta neste tipo de indústria de um separador magnético, que classificasse as partículas magnéticas à medida que elas fossem separadas. Tendo este objectivo em vista desenvolveu-se um novo separador-classificador magnético que consegue obter uma classificação diferencial das partículas magnéticas (Augusto et al., 2002a, b), aliada a uma separação magnética, numa mesma máquina e com uma só passagem.

\section{O NOVO SEPARADOR-CLASSIFICADOR MAGNÉTICO}

Na Figura 3 apresenta-se uma representação esquemática do design do novo separador-classificador magnético.

A teoria suporte do novo aparelho pode ser encontrada em Augusto (2001a), ou em Augusto et al (prep-a,b) e detalhes sobre o design (entre outros) em Augusto (2001b). Aqui será apenas dada uma descrição breve do funcionamento do separador-classificador. Será analisado o caso mais complicado e geral que é o do corpo em rotação. 


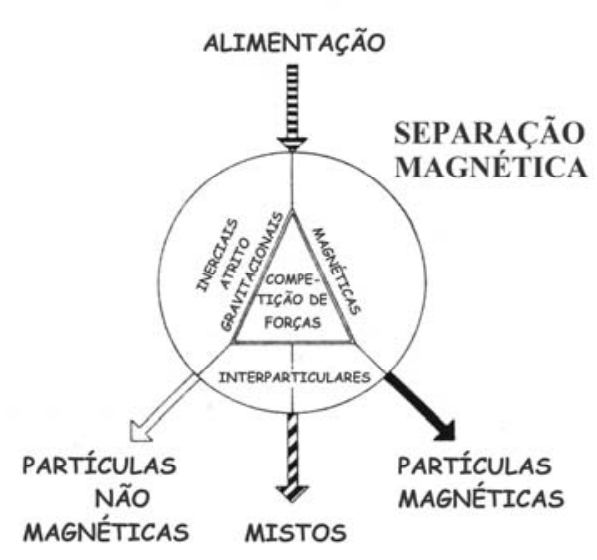

a)

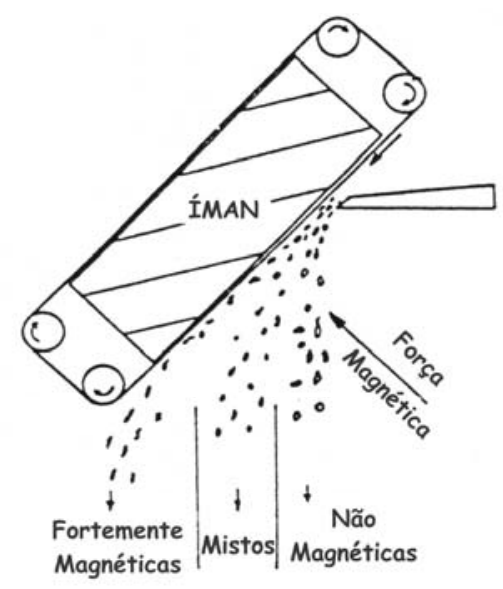

c)

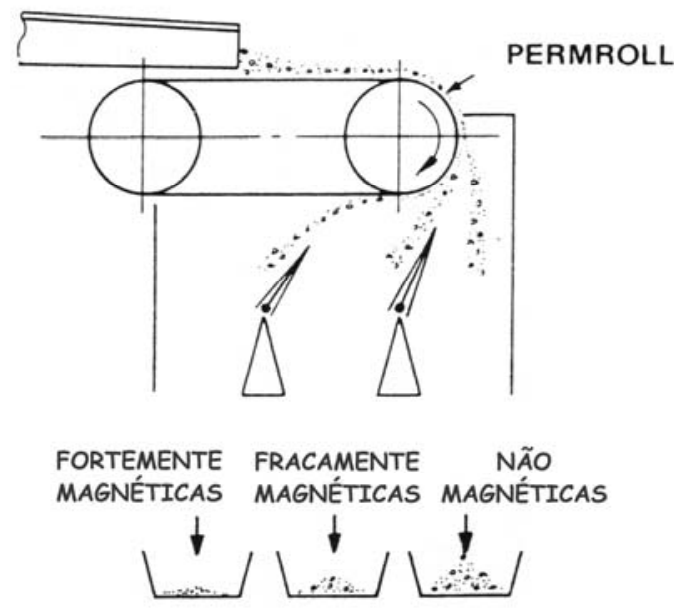

b)

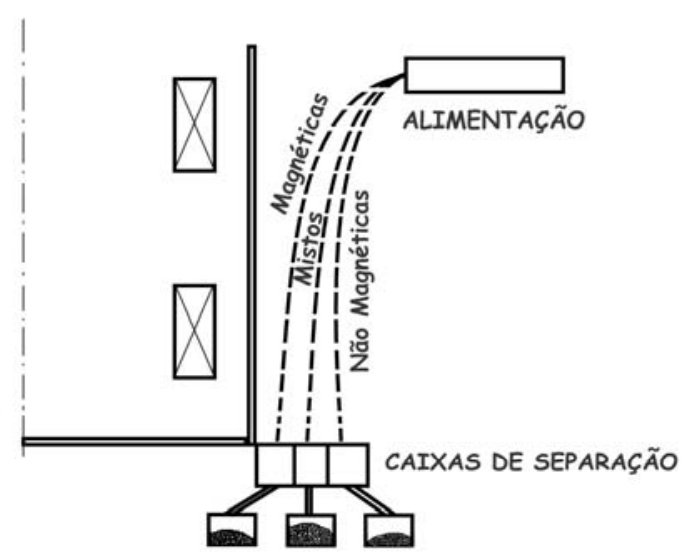

d)

Figura 1 - Processos de separação típicos (baseado em Augusto e Martins (2000a)): a) diagrama processual típico; b) operação de um separador magnético Permroll; c) separação magnética de queda livre; d) separação magnética de gradiente livre. 


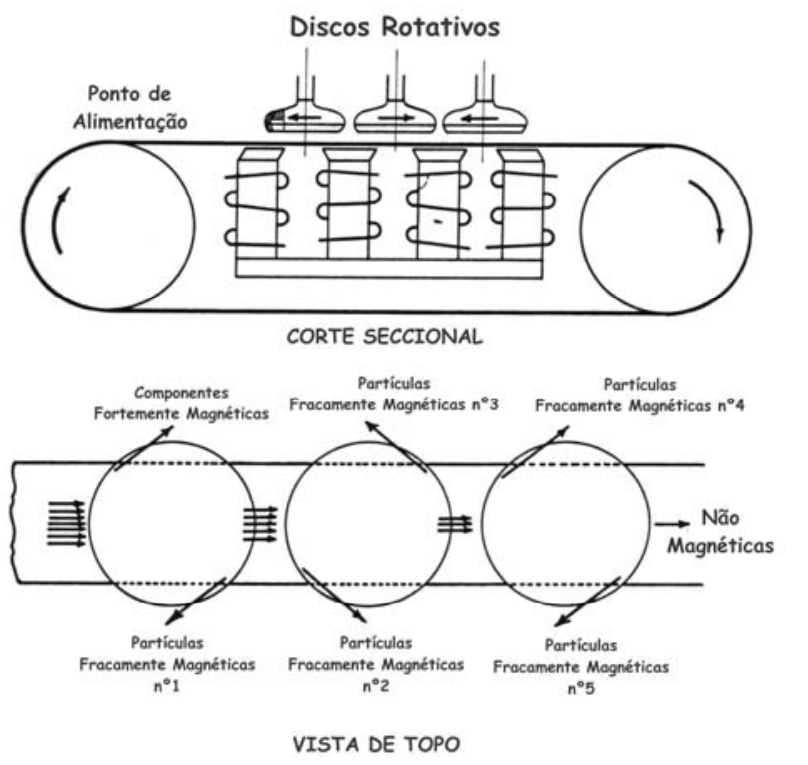

a)

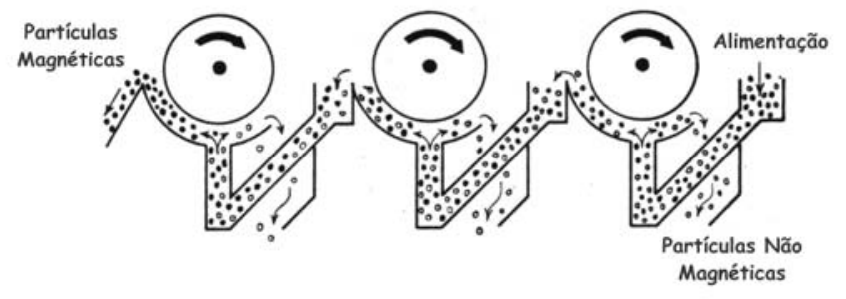

b)

Figura 2 - Processos de separação magnética em cascata clássicos (baseado em Augusto e Martins (2000a)): a) separador de disco rotativo (via seca); b) separadores de tambor em contracorrente (via húmida).

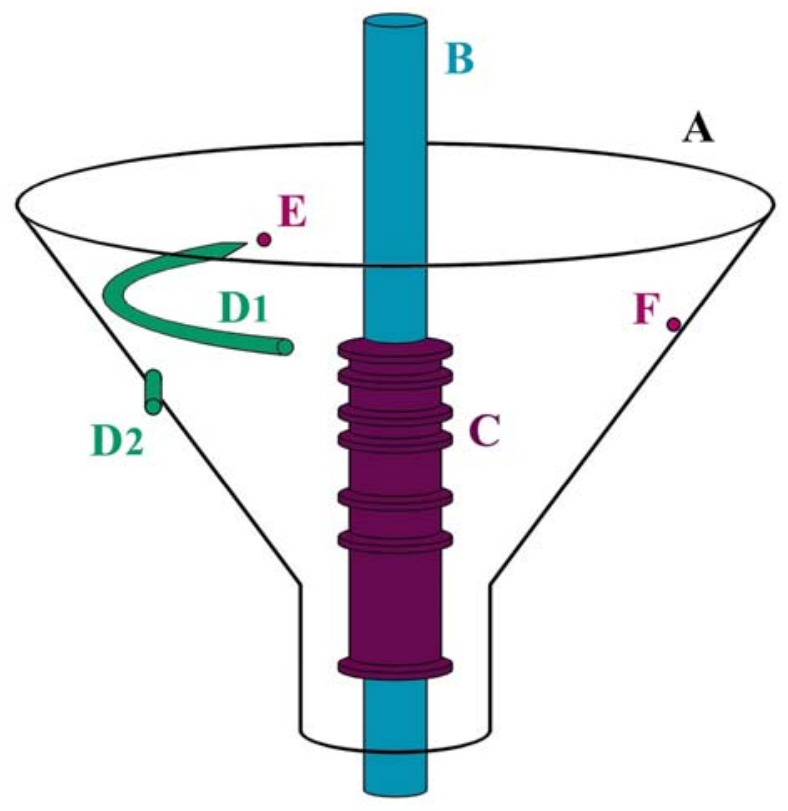

Figura 3 - Representação esquemática do novo separador-classificador magnético (de Augusto e Martins (1999)): A - corpo com a forma de funil (que pode rodar ou não); B - cabo supercondutor; $C$ - colector central desenhado para recolher as partículas magnéticas por classes; D1 e D2 - sistemas de alimentação para a configuração de corpo em rotação e estático, respectivamente; $\mathrm{E}$ - partícula alimentada a um raio inicial ri ; F- partícula magnética. 
O pormenor fundamental é a conjunção entre a configuração geométrica de funil apresentada pelo corpo (estático, ou em rotação), com a configuração de campo magnético concêntrico radial provocada pelo cabo supercondutor. Devido a esta conjunção as partículas magnéticas que são injectadas tangencialmente pela alimentação na superfície do corpo do separador-classificador, sofrerão um força magnética com sentido inverso ao da força centrífuga actuante nas mesmas, uma vez que a força magnética pode ser definida por Stradling (1993),

$$
F_{m}=\frac{m \cdot \chi}{\mu_{0}} \cdot B \nabla B
$$

(válida para partículas pequenas paramagnéticas num meio com susceptibilidade magnética desprezável).

Deste modo as partículas para as quais a susceptibilidade magnética é suficiente para fazer com que a força magnética por elas sentida ultrapasse em magnitude a sua força centrifuga, em vez de subirem na superfície com o resto das partículas, descerão sobre a mesma, obtendo-se deste modo uma primeira separação, uma vez que o valor desta susceptibilidade crítica pode ser escolhido previamente Augusto (2001a).

Ora, no seu movimento descendente, as partículas magnéticas irão aproximar-se gradualmente do centro do separador, sofrendo assim uma força magnética gradualmente superior. Esta força magnética na maioria dos casos e a um certo raio atingirá um valor tal que fará com que a partícula “levante voo” em direcção ao colector central. Da equação (1) apercebemo-nos que as partículas magnéticas que apresentem maior susceptibilidade magnética sentirão esta força magnética crítica num raio superior às restantes, partindo assim em direcção ao centro de um ponto mais longínquo do que as restantes, e chegando deste modo ao colector central a um nível mais elevado. Desenhando assim devidamente um colector central podemos recolher as partículas por classes de susceptibilidade magnética Augusto (2001b).

As partículas magnéticas cuja susceptibilidade magnética seja elevada o suficiente para descerem sobre a superfície do corpo do separador-classificador, mas não seja elevada o suficiente para sofrerem a força magnética crítica cairão num colector de base central.

\section{ALGUMAS APLICAÇÕES PRÁTICAS POSSÍVEIS}

Existem várias aplicações práticas possíveis para este separador-classificador. Iremos no entanto destacar duas pela sua aplicação directa e por constituírem dois casos práticos extremamente elucidativos.

\subsection{Classificação de minérios segundo a sua natureza (constituição química) - selectividade}

Vamos supor que temos um alimentação constituída por diversos minérios entre os quais volframite, hematite, cromite e rodonite (todos 100\% puros), sendo o resto da alimentação partículas ferromagnéticas, paramagnéticas ou diamagnéticas cujas susceptibilidades magnéticas são mais baixas que $3.8 \mathrm{E}-7 \mathrm{~m}^{3} / \mathrm{kg}$ ou maiores que $2.7 \mathrm{E}-6$ 
$\mathrm{m}^{3} / \mathrm{kg}$. Pretendemos então separar as quatro espécies paramagnéticas apontadas umas das outras e do resto das partículas presentes na alimentação. Utilizando então o novo separador-classificador com os parâmetros ajustados pelos valores apresentados em Augusto e Martins (2002b), obteríamos as trajectórias apresentadas na Figura 4.

Na Figura 5 apresenta-se um esquema genérico da classificação de minérios segundo a sua natureza (constituição química) utilizando o novo separador-classificador magnético.

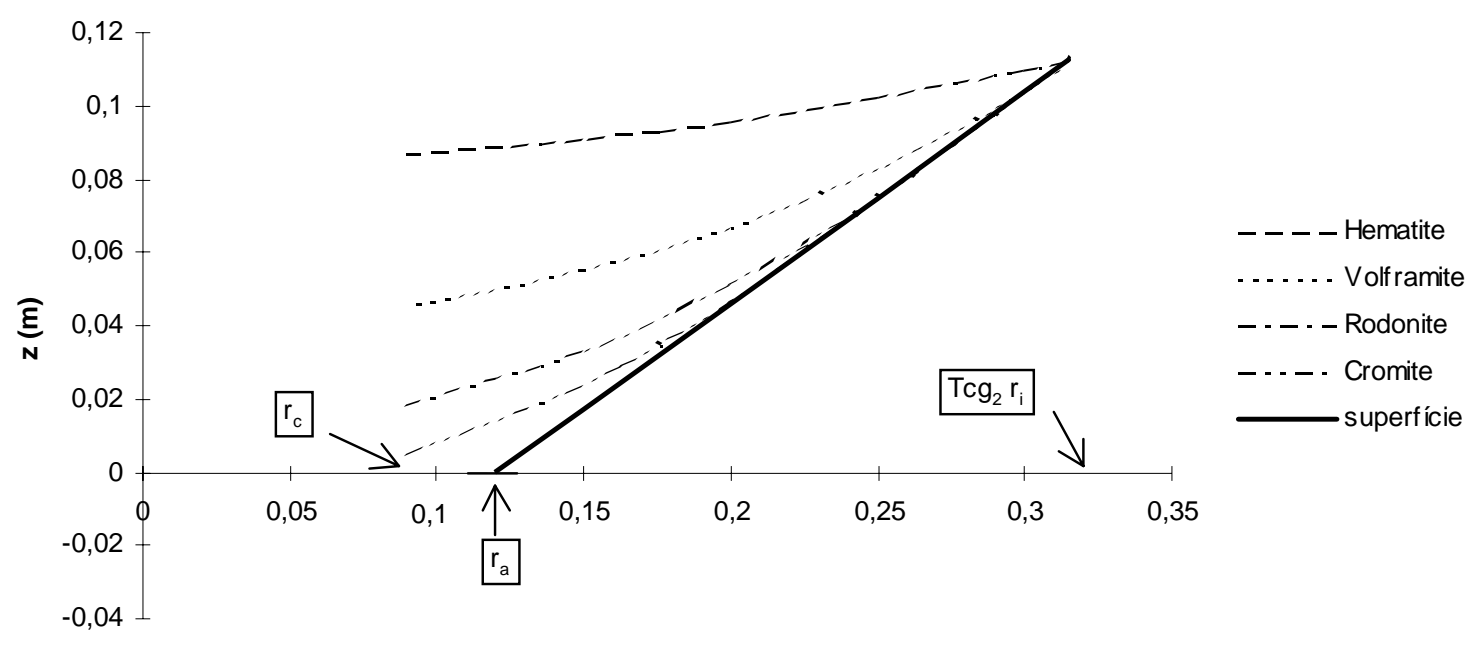

$r(m)$

Figura 4 - Simulações das trajectórias seguidas pelas partículas de diferentes minérios paramagnéticos.

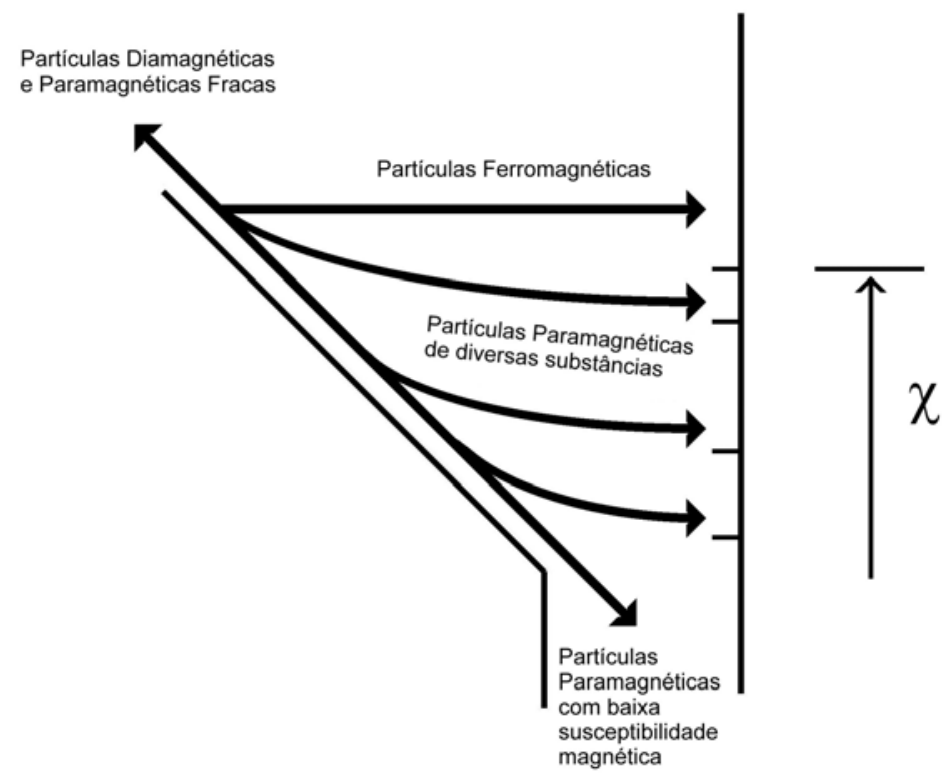

Figura 5 - Representação esquemática das trajectórias seguidas por todos os tipos de partículas alimentadas ao sistema (baseado em Augusto e Martins (2002b)). 


\subsection{Classificação de minérios segundo o seu grau de pureza}

Vamos supor que temos um alimentação constituída por diversos minérios entre os quais volframite em variados graus de pureza, sendo o resto da alimentação partículas ferromagnéticas, paramagnéticas ou diamagnéticas cujas susceptibilidades magnéticas são mais baixas que $4 \mathrm{E}-7 \mathrm{~m}^{3} / \mathrm{kg}$ ou maiores que $1.2 \mathrm{E}-6 \mathrm{~m}^{3} / \mathrm{kg}$. Pretendemos então separar e classificar a volframite consoante o seu grau de pureza, entre os 33.23\% e 100\%. Utilizando então o novo separador-classificador com os parâmetros ajustados pelos valores apresentados em Augusto e Martins (2002b), obteríamos as trajectórias apresentadas na Figura 6.

Na Figura 7 apresenta-se um esquema genérico da classificação de minérios segundo o seu grau de pureza utilizando o novo separador-classificador magnético.

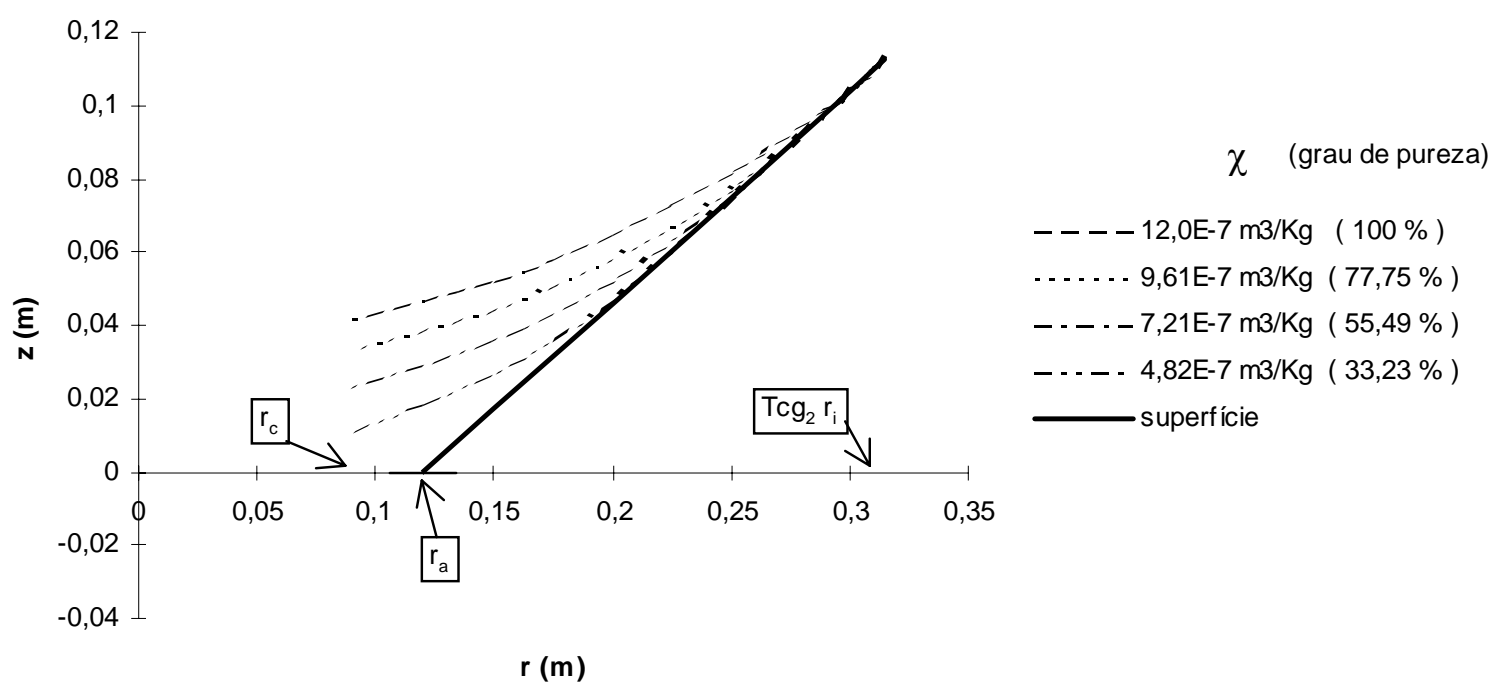

Figura 6 - Simulações das trajectórias seguidas pelas partículas de volframite a diferentes graus de pureza.

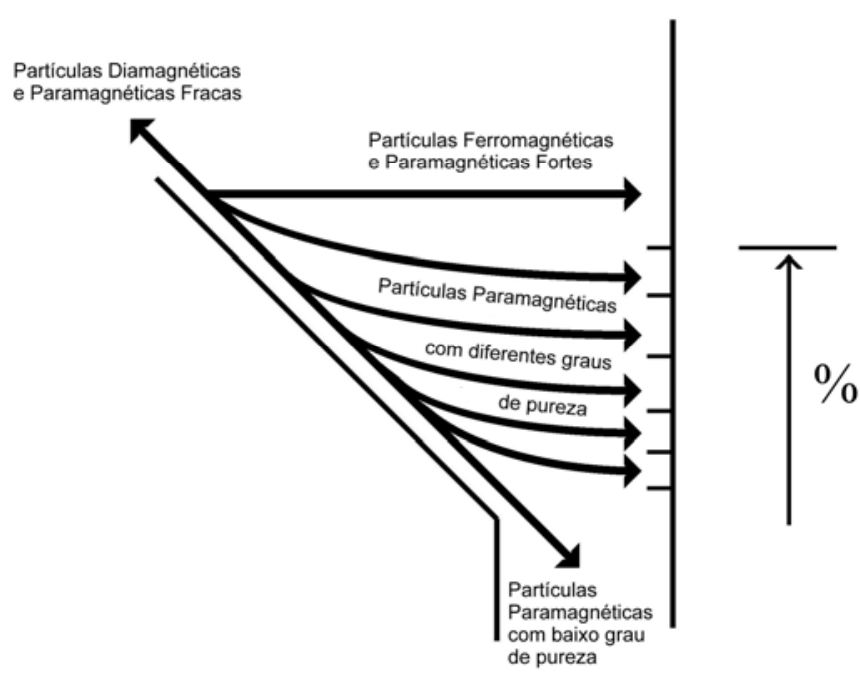

Figura 7 - Representação esquemática das trajectórias seguidas por todos os tipos de partículas alimentadas ao sistema (baseado em Augusto e Martins (2002b)). 


\section{DISCUSSÃO E CONCLUSÕES}

O sector da separação magnética embora bem desenvolvido apresenta uma deficiência a nível da classificação dos minérios magnéticos de acordo com a sua susceptibilidade magnética. O novo separador-classificador por nós desenvolvido e aqui apresentado, preenche esta lacuna, aliando ainda à separação magnética uma classificação diferencial das partículas e utilizando para o efeito o mesmo aparelho, sendo ainda apenas necessária uma passagem do material pelo mesmo.

Deste modo providenciar-se-ão reduções de custos a nível global, e ainda particularmente a nível de solventes, no caso da separação-classificação das partículas magnéticas por grau de pureza. São diversas as possíveis aplicações industriais futuras deste novo aparelho, embora apenas tenham sido analisadas duas das mais imediatas.

\section{REFERÊNCIAS BIBLIOGRÁFICAS}

Augusto, P.A. Um novo separador e classificador magnético. Tese de Doutoramento, Faculdade de Engenharia da Universidade do Porto, Porto, Portugal, 607 p., 2001a.

Augusto, P.A. Separador e classificador magnético. Patente $n^{0} 102326$ (PCT submetida), 2001b.

Augusto, P.A., Augusto, P. \& Castelo-Grande, T. Magnetic Classification. Minerals Engineering 15:1-2, p.35-43, 2002a.

Augusto, P.A., Castelo-Grande, T. \& Augusto, P. Magnetic Classification: a new direction for magnetic separation. Industrial Minerals 423, p.52-57, Dec 2002b (invited paper).

Augusto, P.A., Castelo-Grande, T., Augusto, P., Barbosa, D. \& Estévez, A. A new magnetic separator and classifier: support theory. (em preparação), prep-a.

Augusto, P.A., Castelo-Grande, T., Augusto, P., Barbosa, D. \& Estévez, A. A new magnetic separator and classifier: simulations and boundary conditions for the manipulated variables. Optimization. (em preparação), prep- $b$.

Augusto, P.A. \& Martins, J.P. A new magnetic separator and classifier: prototype design. Minerals Engineering 12:7, p.799-807, 1999.

Augusto, P.A. \& Martins, J.P. Innovation features of a new magnetic separator and classifier. Mineral Processing and Extractive Metallurgy Review, p.1-17, 2000a.

Augusto, P.A. \& Martins, J.P. A new magnetic separator and classifier: case study. Proceedings of the XXI IMPC, Volume C, Chapter 7, p.9-14, First Edition, Developments in Mineral Processing Serie n.13, Elsevier Science B.V., 2000b.

Stradling, A.W. The physics of open-gradient dry magnetic separation. International Journal of Mineral Processing, 39, p.1-18, 1993. 